\title{
Biomonitoring of nutritional acrylamide intake by consumers without dietary preferences as compared to vegans
}

\author{
Katharina Goerke ${ }^{1} \cdot$ Meike Ruenz $^{1} \cdot$ Alfonso Lampen $^{2} \cdot$ Klaus Abraham $^{2} \cdot$ Tamara Bakuradze $^{1} \cdot$ Gerhard Eisenbrand $^{1}$. \\ Elke Richling ${ }^{1}$ (D)
}

Received: 28 September 2018 / Accepted: 13 February 2019 / Published online: 20 February 2019

(c) The Author(s) 2019

\begin{abstract}
Acrylamide (AA) is a heat-induced food contaminant considered as genotoxic carcinogen. The present study investigated the influence of nutritional and lifestyle preferences on human AA exposure. A 10-day human study was performed with ten volunteers without nutritional preferences (omnivores) and ten vegans. Volunteers self-reported their daily routine and dietary habits. Overall mean AA intake, calculated from contents of diet duplicates, was $0.32 \pm 0.19 \mu \mathrm{g} / \mathrm{kg}$ body weight (bw)/day with marked inter-day and inter-volunteer variabilities. Vegans ingested more AA $(0.38 \pm 0.23 \mu \mathrm{g} / \mathrm{kg}$ bw/day $)$ than omnivore volunteers without dietary restrictions $(0.26 \pm 0.10 \mu \mathrm{g} / \mathrm{kg}$ bw/day). Excretion kinetics of urinary AA-related mercapturic acids $N$-acetyl-S-(2-carbamoylethyl)-L-cysteine and $N$-acetyl-S-(2-hydroxy-2-carbamoylethyl)-L-cysteine were essentially concordant with the respective dietary AA intake. Disproportionately enhanced AA-related biomarker excretion could be traced back to reportedly inadvertent, passive exposure to tobacco and/or fire smoke, as evidenced by the respective urinary exposure biomarkers, cotinine and $N$-acetyl-S-(2-cyanoethyl)-L-cysteine. Although the study is based on the comparison of small volunteer groups, the results confirm the association of AA exposure biomarkers with documented dietary preferences and lifestyle factors. Some additional contribution of endogenous background AA exposure was demonstrated individually. Disproportionately enhanced AA exposure is suggested to result from passive exposure to tobacco and/or barbecue smoke.
\end{abstract}

Keywords Acrylamide $\cdot$ Biomonitoring $\cdot$ Dietary exposure $\cdot$ Human study $\cdot$ Mercapturic acids $\cdot$ Vegan diet

\section{Introduction}

Acrylamide (AA) is a process-related contaminant that forms during heating of food from asparagine and reducing sugars in the so-called Maillard reaction. AA levels in food strongly depend on the type, temperature and duration of processing, such as frying or baking. Especially foods rich in

Electronic supplementary material The online version of this article (https://doi.org/10.1007/s00204-019-02412-x) contains supplementary material, which is available to authorized users.

Elke Richling

richling@chemie.uni-kl.de

1 Division of Food Chemistry and Toxicology, Department of Chemistry, Technische Universitaet Kaiserslautern, Erwin-Schroedinger-Straße 52, 67663 Kaiserslautern, Germany

2 Department of Food Safety, German Federal Institute for Risk Assessment (BfR), Max-Dohrn-Straße 8-10, 10589 Berlin, Germany carbohydrates are prone to increased AA contamination as a consequence of heat treatment. (Rydberg et al. 2003; Jackson and Al-Taher 2005; Zyzak et al. 2003; Mottram et al. 2002). These include fried potato products (mean of $332 \mu \mathrm{g} / \mathrm{kg}$ ), potato crisps $(580 \mu \mathrm{g} / \mathrm{kg})$, certain breakfast cereals $(113 \mu \mathrm{g} /$ $\mathrm{kg})$, biscuits, crackers, crisp bread or similar $(264 \mu \mathrm{g} / \mathrm{kg})$ and roasted coffee $(317 \mu \mathrm{g} / \mathrm{kg})$. Dietary exposure estimates for adults (aged 18-65 years) range from 0.4 to $0.6 \mu \mathrm{g} / \mathrm{kg}$ body weight (bw)/day (mean) and $0.8-1.3 \mu \mathrm{g} / \mathrm{kg}$ bw/day (95th percentile), respectively (EFSA 2015). In addition to foods of industrial origin, home cooking is contributing to an as yet largely unexplored extent to the AA overall exposure. Finally, AA also arises by combustion processes and therefore is present in tobacco smoke (Mojska et al. 2016). AA is considered a genotoxic carcinogen undergoing CYP450 2E1 mediated epoxidation in the liver to 2,3-epoxypropanamide, an alkylating electrophile also called glycidamide (GA). Both, AA and GA, are predominantly conjugated with glutathione (GSH) and further metabolized to mercapturic acids (MA) like $N$-acetyl-S-(2-carbamoylethyl)-L-cysteine 
(AAMA), derived from AA, and $N$-acetyl-S-(2-hydroxy2-carbamoylethyl)-L-cysteine (GAMA), derived from GA (Fennell et al. 2005; Watzek et al. 2012b; Boettcher et al. 2006). AAMA and GAMA are widely utilized as short-term biomarkers of exposure, because they are excreted within about 3 days following exposure (Goempel et al. 2017). In contrast, adducts to the $N$-terminal valine of hemoglobin $(\mathrm{Hb})$ are conceived as biomarkers reflecting cumulative exposure over the lifetime of red blood cells, known to be completely replaced within about 4 months in humans (Vikström et al. 2012).

The aim of the present study was to investigate the potential influence of nutritional consumption preferences on dietary AA exposure. To this end, regular home cooked food of volunteers without specific dietary regimen was analyzed in comparison to that of vegans by determination of AA contents in representative aliquots of self-prepared diet duplicates. Subsequently, the respective total urines excreted within $12 \mathrm{~h}$ time periods were monitored for AA-related mercapturic acids as urinary AA exposure biomarkers. All volunteers were unrestricted and free-living individuals, whose normal lifestyle habits were not interfered with by any guideline given due to study participation. In addition to AA-related biomarkers, biomarkers of inadvertent smoke exposure by tobacco or open fire (e.g., connected with outdoor barbecuing) were monitored as well. This was achieved by monitoring urinary cotinine, the major metabolite of nicotine present in tobacco smoke, and urinary $N$-acetyl-S-(2cyanoethyl)-L-cysteine (CYMA), a biomarker supposed to reflect exposure to acrylonitrile. The latter is a basic chemical used, e.g., in polymer synthesis that is also generated by combustion of organic material, e.g., at an open fire or barbecue, and is also generated during tobacco smoking. Inhalative exposure to acrylonitrile can be monitored by urinary CYMA quantification (Bramer and Kallungal 2003; Minet et al. 2011; Jakubowski et al. 1987; Goniewicz et al. 2017).

\section{Materials and methods}

\section{Study design}

Twenty healthy non-smoking volunteers of Caucasian origin were recruited and allocated to two different groups: ten (five male, five female) who did not declare any dietary preferences, hereinafter called omnivores, and ten (five male, five female) who declared to follow a vegan lifestyle by abstaining from consumption of animal products. Exclusion criteria were: age $<20$ or $>50$ years, body mass index $(\mathrm{BMI})<19$ or $>25 \mathrm{~kg} / \mathrm{m}^{2}$, smoking or tobacco consumption, metabolic disorders, performing competitive sports, being under medication or taking dietary supplements, participation in other studies, regular blood donation, and pregnancy in women.
All volunteers gave their informed consent. A medical check was performed prior to study start to determine bw, height, BMI, blood pressure, and clinical parameters including general blood cell counts as well as creatinine, glomerular filtration rate, $\gamma$-glutamyl transferase, and C-reactive protein. Volunteers were aged $26 \pm 5$ years and were of good health status with a BMI of $22 \pm 2 \mathrm{~kg} / \mathrm{m}^{2}$.

During the 10-day study all volunteers maintained their daily living routine as well as their dietary habits. All consumed meals and beverages were protocolled (time, portion size) by the volunteers and representative aliquots $(\geq 50 \mathrm{~g}$, judgement by volunteers themselves) were delivered daily to the study location, where they were homogenized immediately and stored at $-20{ }^{\circ} \mathrm{C}$ until analysis. Duplicates of meals consumed in the university canteen were provided by the canteen staff, homogenized in the lab and stored at $-20{ }^{\circ} \mathrm{C}$ until analysis. Volunteers preparing their own meals at home were instructed in detail prior to study start and trained how to generate aliquots representative for their meals as closely as possible by replicating portion size and constituent distribution. The study was carried out during summer time (June-August 2015). Each study day started at 8.00 a.m. $(0 \mathrm{~h})$. Total urine was collected during the whole study in two sequential $12 \mathrm{~h}$ intervals per day ( 8 a.m. to 8 p.m. respective 8 p.m. to 8 a.m.). Urine was delivered daily to the study location, weighed and stored at $-20{ }^{\circ} \mathrm{C}$ until analysis of AAMA and GAMA. Additionally, in 16 of 20 volunteers consenting to a study amendment urinary CYMA and cotinine were analyzed. After 9 days one volunteer (vegan-male-5) quit the study due to a clinical surgery. All volunteers were asked to retrospectively fill out activity protocols approximately 1 year after the study to obtain information on inadvertent exogenous AA sources they may have been potentially exposed to, like open fire, barbecuing, and/or second-hand smoke.

\section{Experimental procedures}

\section{Chemicals}

AA was purchased from Sigma Aldrich (St. Louis, USA). Deuterium-labeled AA ( $\mathrm{D}_{3}$-AA), $N$-acetyl- $S$ (carbamoylethyl)-L-cysteine (AAMA), $N$-acetyl- $S$ (1-carbamoyl-2-hydroxyethyl)-L-cysteine (GAMA), $N$-acetyl-S-(2-cyanoethyl)-L-cysteine (CYMA), ( \pm )-1-(methyl)-5-(2-pyridinyl)-2-pyrrolidinone (raccotinine) as well as the deuterium-labeled analogues $N$-(acetyl- $\mathrm{D}_{3}$ )-S-(carbamoylethyl)-L-cysteine ( $\mathrm{D}_{3}$-AAMA), $\mathrm{N}$-(acetyl- $\mathrm{D}_{3}$ )-S-(1-carbamoyl-2-hydroxyethyl)-L-cysteine $\left(\mathrm{D}_{3} \text {-GAMA) and }( \pm)-1 \text {-(Methyl-D }\right)_{3}$-5-(2-pyridinyl)2-pyrrolidinone $\left(\mathrm{D}_{3}\right.$-cotinine $)$ were obtained from Toronto Research Chemicals (Toronto, Canada). All HPLC solvents were LC-MS grade, other reagents were of analytical grade. 


\section{Acrylamide contents of food}

Acrylamide contents of relevant food samples were determined in duplicate by a stable isotope dilution method (SIDA) as recently described (Goempel et al. 2017). In short, food samples were extracted with water using $250 \mathrm{ng} \mathrm{D}_{3}$-AA as internal standard for all food samples, then purified via solid phase extraction (SPE) using Isolute ${ }^{\circledR} \mathrm{ENV}+$ columns (Biotage, Uppsala, Sweden), concentrated and measured via high performance liquid chromatography tandem mass spectrometry (HPLC-MS/MS) (Agilent 1290 Infinity Series HPLC, Waldbronn, Germany, coupled to an AB Sciex QTrap 5500, Darmstadt, Germany) using positive electrospray ionization and the multiple reaction monitoring (MRM) mode.

\section{Urine analysis [mercapturic acids, cotinine, urinary creatinine (ucr)]}

Assuming a density of $1 \mathrm{~kg} / \mathrm{L}$, total urine weight was converted into total urine volume. Urinary creatinine (ucr) was determined in duplicate using an ucr assay kit purchased from Cayman Chemical Company (Ann Arbor, USA) following the manufacturer's instructions. Urinary MA were expressed as amounts per excreted total urine volume and normalized to ucr. Determination of AAMA and GAMA followed the SIDA method as published before (Goempel et al. 2017). In brief, urine samples (1 $\mathrm{mL}$ each) were purified via SPE using Isolute ${ }^{\circledR}$ ENV+ columns (Biotage, Uppsala, Sweden) and diluted 1:4 for analysis via UHPLC-ESI-MS/ MS (Agilent 1290 Infinity Series HPLC + AB Sciex QTrap 5500). Measurements were performed in the scheduled MRM mode.

Cotinine and CYMA were determined in the SPE eluates prepared to quantitate AAMA and GAMA. To that end, these eluates were further diluted (1:4) with a $\mathrm{D}_{3}$-cotinine solution, resulting in a final $\mathrm{D}_{3}$-cotinine concentration of $3 \mathrm{ng} / \mathrm{mL}$. Since adequately pure $\mathrm{D}_{3}$-CYMA was not available CYMA was estimated by external calibration. A calibration curve was established each time immediately before analysis of the urine samples of each volunteer. Separation of cotinine and CYMA was achieved by reversed phase chromatography using an Agilent Zorbax Eclipse XDB-C18 column $(50 \times 4.6 \mathrm{~mm}, 1.8 \mu \mathrm{m})$ equipped with the corresponding UHPLC guard column. Gradient elution was carried out with $0.1 \%$ aqueous acetic acid (A) and acetonitrile (B) at a flow rate of $600 \mu \mathrm{L} / \mathrm{min}$. After $0.5 \mathrm{~min}$ at $4 \% \mathrm{~B}$, it was increased to $9 \%$ within $3.3 \mathrm{~min}$, followed by a reconditioning step. MS/MS detection was based on the shuttled MRM mode switching ionization from positive (cotinine) to negative (CYMA) after 3 min (MS parameters see Supporting Information Table S1). Absolute SPE recovery rates were 82-99\% for cotinine and $77-87 \%$ for CYMA (measured with four different concentrations each). Limits of detection
(LOD) and limits of quantification (LOQ) were $20 \mathrm{pg} / \mathrm{mL}$ and $75 \mathrm{pg} / \mathrm{mL}$ for cotinine as well as $15 \mathrm{pg} / \mathrm{mL}$ and $50 \mathrm{pg} /$ $\mathrm{mL}$ for CYMA.

\section{Data analysis}

MS data were processed by Analyst 1.6.1 and Multiquant 2.1.1 Software (AB Sciex). Data analysis and plots were set up with Excel 2013 (Microsoft, Washington, USA) and Origin ${ }^{\circledR} 2018$ Software (Origin Lab Corporation, Northampton, USA), respectively. Normal distribution was checked by the Anderson-Darling test. When normality could not be rejected, a two-sided, unpaired $t$ test was used to compare groups.

\section{Results}

A 10-day human study was performed with two groups of healthy volunteers $(n=10$ each) to monitor dietary AA intake via analysis of diet duplicates prepared by the volunteers. One group did not report any dietary preferences (omnivores), the other declared to follow a vegan lifestyle. In parallel, urinary excretion of the AA exposure biomarkers AAMA and GAMA was monitored. In a subgroup $(n=16)$ consenting to a study amendment, (tobacco) smoke-related biomarkers cotinine and CYMA were monitored.

During the study all 20 volunteers provided self-prepared aliquots of the consumed meals for AA analysis. The AA intake was calculated individually for each study day and each individual volunteer by a middle bound approach as described by the European Food Safety Authority (EFSA) in 2015. Therefore, all AA values < LOD (ranging, in dependence of the matrix, from 0.3 to $24 \mu \mathrm{g} / \mathrm{kg}$ ) were set to 0 , whereas values $>$ LOD and $<$ LOQ were set to half the LOQ (LOQ range 1.1-36 $\mu \mathrm{g} / \mathrm{kg}$ ). Only food expected to have quantifiable AA contents was analyzed. Foods and beverages that had not been treated with a process prone to bring about noteworthy AA contamination, such as water, soft drinks, juices, milk and milk products, raw fruits and fresh vegetables were presumed not to contain appreciable AA contamination, and were therefore excluded from analysis (EFSA 2015; Ruenz et al. 2016). Grouped AA contents of typical food items are given in Table 1.

A broad range of beverage and food samples was analyzed. As shown in Table 1, food items processed at $>120{ }^{\circ} \mathrm{C}$ revealed high AA contents (mean of $119 \mu \mathrm{g} / \mathrm{kg}$, maximum $289 \mu \mathrm{g} / \mathrm{kg}$ ). Commercially available snack products such as crisps and snacks, toasted tomato bread and pretzel sticks were found to contain the highest amounts of AA with a mean of $147 \mu \mathrm{g} / \mathrm{kg}$ (maximum $481 \mu \mathrm{g} / \mathrm{kg}$ in a crisps sample). Meals consisting of meat and heat-processed products were mainly from the canteen of the university, 
Table 1 Mean, minimum $(\min )$ and maximum $(\max )$ acrylamide contents of different food categories (with $n$ food samples each) consumed during the human study by volunteers

\begin{tabular}{|c|c|c|c|c|}
\hline & \multirow[t]{2}{*}{$n$} & \multicolumn{3}{|c|}{$\begin{array}{l}\text { Acrylamide con- } \\
\text { tent }(\mu \mathrm{g} / \mathrm{kg})\end{array}$} \\
\hline & & Mean & Min & $\operatorname{Max}$ \\
\hline Heat processed potato products ${ }^{\mathrm{a}}$ & 18 & 119 & 9 & 289 \\
\hline Crisps and snacks & 11 & 147 & 15 & 481 \\
\hline $\begin{array}{l}\text { Meals with meat and highly heated } \\
\text { products }^{\mathrm{b}}\end{array}$ & 8 & 81 & 29 & 202 \\
\hline Meat surrogates (e.g., tofu) \pm side dishes & 12 & 50 & 15 & 100 \\
\hline Vegetarian meals & 7 & 24 & 5 & 48 \\
\hline Bread and sandwiches $^{c}$ & 26 & 50 & 8 & 210 \\
\hline Bakery goods and biscuits & 22 & 43 & 6 & 241 \\
\hline Sweets $^{\mathrm{d}}$ & 11 & 84 & 9 & 160 \\
\hline Coffee beverages ( \pm milk) & 12 & 8 & 4 & 22 \\
\hline
\end{tabular}

${ }^{a}$ E.g., French fries, croquettes

${ }^{b}$ Mostly meals from the university's canteen like French fries with schnitzel

${ }^{c}$ Bread (rolls) with or without topping

${ }^{\mathrm{d}}$ E.g., ice cream, chocolate

as many volunteers were students. Mean AA contents in these meals were $81 \mu \mathrm{g} / \mathrm{kg}$. In a home cooked meal containing meat (not specified) with potatoes a maximum value of $202 \mu \mathrm{g} / \mathrm{kg}$ was measured.

Meat surrogates like tofu or seitan with or without side dishes like gnocchi showed lower mean AA contents $(50 \mu \mathrm{g} /$ $\mathrm{kg}$ ) with a maximum of $100 \mu \mathrm{g} / \mathrm{kg}$ in a grilled tofu sausage. Vegetarian meals mainly consisting of vegetables showed lower mean AA contents with a maximum of $48 \mu \mathrm{g} / \mathrm{kg}$. Samples of bread (rolls) with or without topping showed an overall average AA content of $50 \mu \mathrm{g} / \mathrm{kg}$ and maximum values of $143 \mu \mathrm{g} / \mathrm{kg}$ and $210 \mu \mathrm{g} / \mathrm{kg}$ in olive and walnut bread, respectively. Bakery goods and biscuits revealed mean AA contents of $43 \mu \mathrm{g} / \mathrm{kg}$ with maximum values of $149 \mu \mathrm{g} / \mathrm{kg}$ and $241 \mu \mathrm{g} / \mathrm{kg}$ in two cake samples. Sweets showed a broad range of AA contents from $9 \mu \mathrm{g} / \mathrm{kg}$ (praline ice cream) to $160 \mu \mathrm{g} / \mathrm{kg}$ (hazelnut waffles). During the study, about half of the volunteers drank coffee with or without milk or sugar (mean of $8 \mu \mathrm{g} / \mathrm{kg}$, maximum $22 \mu \mathrm{g} / \mathrm{kg}$ in an espresso).

Daily AA intake of each volunteer at the respective study day was estimated, based on the quantified AA contents in the consumed foods with the self-protocolled portion size and ingestion time (see Supporting Information Table S2). During the 10-day study, mean dietary AA exposure of all volunteers was $299 \mathrm{nmol} /$ day $(0.32 \pm 0.19 \mu \mathrm{g} / \mathrm{kg}$ bw/day $)$ (see Table 2), with marked inter-day and inter-volunteer variabilities (see Fig. 1a). On some study days, AA was not found $(<$ LOD) in any consumed meals, consequently dietary intake of the corresponding volunteer was set to 0 nmol. Male volunteers showed a higher absolute AA intake
Table 2 Mean and absolute maximum (max) daily acrylamide intake of all volunteers (vegans and omnivores) during the 10-day study and averaged sum of the whole study

\begin{tabular}{|c|c|c|c|c|c|}
\hline & \multirow[t]{3}{*}{$n$} & \multicolumn{4}{|c|}{ Acrylamide intake } \\
\hline & & \multicolumn{2}{|c|}{$\mu \mathrm{g} / \mathrm{kg}$ bw/day } & \multicolumn{2}{|c|}{$\mathrm{nmol} / \mathrm{day}$} \\
\hline & & Mean & Max & Mean & $\operatorname{Max}$ \\
\hline All volunteers & 20 & 0.32 & 2.51 & 299 & 2200 \\
\hline Men & 10 & 0.29 & 1.87 & 323 & 2200 \\
\hline Women & 10 & 0.35 & 2.51 & 276 & 2112 \\
\hline Omnivores & 10 & 0.26 & 1.58 & 244 & 1097 \\
\hline Male omnivores & 5 & 0.21 & 0.93 & 237 & 1091 \\
\hline Female omnivores & 5 & 0.33 & 1.58 & 264 & 1097 \\
\hline Vegans & 10 & 0.38 & 2.51 & 355 & 2200 \\
\hline Male vegans & 5 & 0.38 & 1.87 & 409 & 2200 \\
\hline Female vegans & 5 & 0.39 & 2.51 & 302 & 2112 \\
\hline
\end{tabular}

$(323 \pm 212 \mathrm{nmol} /$ day $)$ than the females $(276 \pm 145 \mathrm{nmol} /$ day), however, on a body weight base, intake was $0.29 \pm 0.18 \mu \mathrm{g} / \mathrm{kg}$ bw/day for males and $0.35 \pm 0.20 \mu \mathrm{g} / \mathrm{kg}$ bw/day for females. Furthermore, vegan volunteers ingested more AA (mean $355 \pm 228 \mathrm{nmol} / \mathrm{day}$ and $0.38 \pm 0.23 \mu \mathrm{g} / \mathrm{kg}$ bw/day, respectively) than the omnivore volunteers (mean $244 \pm 90 \mathrm{nmol} /$ day and $0.26 \pm 0.10 \mu \mathrm{g} / \mathrm{kg}$ bw/day, respectively), yet this difference was not significant ( $p \geq 0.18)$.

Foods consumed were categorized into groups with respect to contribution to dietary AA intake (see Supporting Information Table S3). In general, frequent ingestion of raw fruits and vegetables (e.g., salad) led to lower AA intake, as compared to heated foods, bread and bakery products or coffee beverages. Meals with heat-processed potato products like French fries and potato crisps led to enhanced AA intake, accounting for $31 \%$ (mean) of the overall AA intake during the study. Heated meals (not deep fried) were consumed more often and regularly than deep-fried potato products, in total accounting for about a quarter of the AA exposure. Such products contributed by about $20 \%$ to the AA exposure of the omnivore volunteers and by $29 \%$ to that of vegan volunteers. Almost all volunteers $(n=18)$ consumed bread, bread rolls or pretzels during the study, accounting for $16 \%$ of the total AA intake of all volunteers, again with a marked difference between omnivores (8\%) and vegans (23\%). Bakery goods and biscuits were mainly consumed by omnivore volunteers, accounting for $15 \%$ of their overall AA intake, in contrast to vegan volunteers (5\%). Furthermore, a mean of $18.4 \pm 10.0 \mu \mathrm{g} / 10$ days AA was ingested via coffee drinking, equivalent to $11 \pm 5 \%$ of the overall AA intake among coffee drinkers (11 out of 20 volunteers).

Results of monitoring the urinary AA exposure biomarkers AAMA and GAMA throughout the whole 10-day study period are given in detail, as sum of two 12-h intervals, in Supporting Information Tables S3 (AAMA) and 
Fig. 1 Overview of a the AA intake and b AAMA excretion during the 10-day study for each volunteer. Volunteer vegan-male-5 quit after 9 days, so data were summarized only for 9 days. Boxes, Perc 25/75, whisker, standard deviation; black squares, mean; dash, median; times symbol, minimum; asterisk, maximum

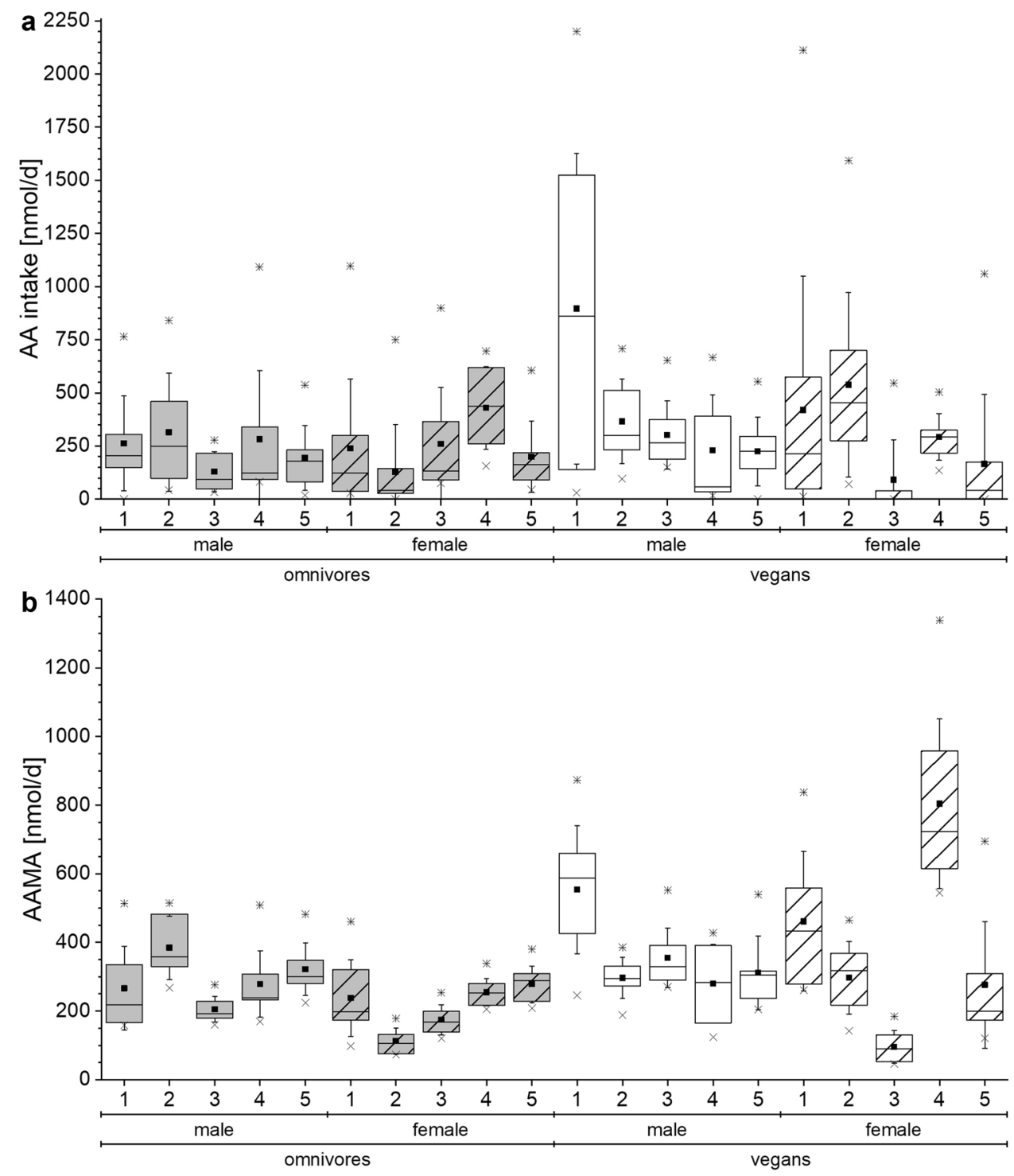

S4 (GAMA). Table 3 gives a group summary. Daily mean excretion of AAMA was $312 \mathrm{nmol} /$ day (46-1338 nmol/day) for all volunteers, that of GAMA as minor metabolite was $45 \mathrm{nmol} /$ day (11-109 nmol/day). In accordance with the dietary AA intake, vegan volunteers excreted more AAMA (374 nmol/day) and GAMA (50 nmol/day) than the omnivore group (251 nmol/day for AAMA and $40 \mathrm{nmol} /$ day for GAMA).

As mentioned, every volunteer kept his/her usual dietary and lifestyle habits. Reflecting the respective individual AA intake (Fig. 1a), AAMA excretion varied strongly from volunteer to volunteer and between days (Fig. 1b).

Individual AA intake and associated biomarker excretion in three volunteers are exemplified in Fig. 2. The omnivorefemale-1 with an unrestricted diet (Fig. 2a) consumed AAcontaining food every study day, resulting in a mean dietary AA exposure of $238 \mathrm{nmol} /$ day $(0.34 \mu \mathrm{g} / \mathrm{kg}$ bw/day). On days
1, 5 and 9, this volunteer ingested relatively high amounts of AA by having lunch at the university canteen with major contribution ( $79 \%$ of total AA intake) by fried potato products (day 1: pork blade steak with French fries and side dishes, $29.2 \mu \mathrm{g} / \mathrm{kg}$ consumed meal; day 5: potato pancakes with apple puree and soup, $152 \mu \mathrm{g} / \mathrm{kg}$ consumed meal; day 9: Swiss cheese salad with French fries and side dishes, $48.4 \mu \mathrm{g} / \mathrm{kg}$ consumed meal). Accordingly, the excretion of AAMA and GAMA reached maximum levels in the overnight urine of day 1 , day 5 , and day 9 . In the intervening time periods with low AA intake values were decreasing within $72 \mathrm{~h}$ (day 9: $48 \mathrm{~h}$ until study end). The excretion of CYMA was very low during the whole study $(0.4 \pm 0.1 \mathrm{nmol} /$ day $)$, levels of cotinine were $<$ LOD.

In contrast, vegan-female-3 (Fig. 2b) consumed barely quantifiable AA amounts on 3 days during the study, resulting in the lowest overall AA intake of all 20 volunteers 
Table 3 Mean and absolute $\operatorname{minima} / \operatorname{maxima}(\min / \max )$ excreted amounts (in nmol/ day) of AAMA ( $N$-acetyl- $S$ - $(2-$ carbamoylethyl)-L-cysteine) and GAMA ( $N$-acetyl- $S$-(2-hydroxy2-carbamoylethyl)-L-cysteine) during the 10-day study

\begin{tabular}{|c|c|c|c|c|c|c|c|}
\hline & \multirow[t]{3}{*}{$n$} & \multicolumn{3}{|c|}{ AAMA excretion } & \multicolumn{3}{|c|}{ GAMA excretion } \\
\hline & & \multicolumn{3}{|c|}{ nmol/day } & \multicolumn{3}{|c|}{$\mathrm{nmol} /$ day } \\
\hline & & Mean & Min & $\operatorname{Max}$ & Mean & Min & $\operatorname{Max}$ \\
\hline All volunteers & 20 & 312 & 46 & 1338 & 45 & 11 & 109 \\
\hline Men & 10 & 325 & 124 & 873 & 52 & 26 & 109 \\
\hline Women & 10 & 299 & 46 & 1338 & 38 & 11 & 103 \\
\hline Omnivores & 10 & 251 & 73 & 514 & 40 & 11 & 85 \\
\hline Male & 5 & 291 & 159 & 514 & 50 & 30 & 85 \\
\hline Female & 5 & 211 & 73 & 460 & 30 & 11 & 50 \\
\hline Vegans & 10 & 374 & 46 & 1338 & 50 & 16 & 109 \\
\hline Male & 5 & 360 & 124 & 873 & 53 & 26 & 109 \\
\hline Female & 5 & 387 & 46 & 1338 & 46 & 16 & 103 \\
\hline
\end{tabular}

$n$ number of volunteers (mean of $91 \mathrm{nmol} /$ day, $0.1 \mu \mathrm{g} / \mathrm{kg}$ bw/day). On day 1 , bread with and without topping was consumed $(9.2$ and $9.7 \mu \mathrm{g} /$ $\mathrm{kg}$, respectively), on day 2 pan-fried vegetables with soy stripes $(66.4 \mu \mathrm{g} / \mathrm{kg})$, and on day 9 pan-fried vegetables $(23.2 \mu \mathrm{g} / \mathrm{kg})$. Apart from that, mainly fresh fruits and smoothies, mixed salads, vegetables, mineral water, and tea were consumed. Associated with this low AA intake, this volunteer had the lowest AAMA excretion with a mean of $96 \mathrm{nmol} /$ day and a range of 46-185 nmol/day (Fig. 1b). AA intake of $546 \mathrm{nmol}(0.6 \mu \mathrm{g} / \mathrm{kg} \mathrm{bw})$ mainly in the early evening of day 2 resulted in a maximum AAMA excretion of $135 \mathrm{nmol} / 12 \mathrm{~h}(240 \mathrm{nmol} / \mathrm{g}$ ucr $)$ in the overnight urine of day 2. In the absence of any further AA intake, urinary MA excretion declined down to a consistent background, with a mean of $51 \pm 5 \mathrm{nmol} /$ day AAMA ( $48 \pm 10 \mathrm{nmol} / \mathrm{g} \mathrm{ucr})$ and $19 \pm 3 \mathrm{nmol} /$ day $(17 \pm 3 \mathrm{nmol} / \mathrm{g}$ ucr $)$ GAMA between days 5-8. No detectable levels of cotinine were measured in the urine of this volunteer, values for CYMA were in the low end area of all volunteers. Of note, the excretion of both MA never reached zero. For example, vegan-female- 3 after 6 days of zero dietary AA intake still exhibited a baseline excretion of $46 \mathrm{nmol} /$ day for AAMA, respectively, $11 \mathrm{nmol} /$ day for GAMA as lowest values. Since noteworthy exogenous AA exposure was absent, urinary cotinine was $<$ LOD and CYMA extremely low $(0.3-0.6 \mathrm{nmol} /$ day $)$, this indicates the level of endogenous background AA exposure in this volunteer.

As a third example, results for vegan-female-4 are presented in Fig. 2c. Surprisingly, this volunteer excreted disproportionately high amounts of MA, with regard to her mean dietary AA intake of $293 \pm 110 \mathrm{nmol} / \mathrm{day}$ $(0.36 \pm 0.14 \mu \mathrm{g} / \mathrm{kg}$ bw/day $)$, throughout the study ranging from 135 to $503 \mathrm{nmol} /$ day $(0.17-0.63 \mu \mathrm{g} / \mathrm{kg}$ bw/day). Consumption of bread with topping, and bakery goods like pretzels, cake, and biscuits accounted for $82 \%$ of her overall AA intake. This volunteer excreted disproportionately high amounts of AAMA and GAMA, with means of $804 \mathrm{nmol} /$ day (545-1338 nmol/day) and $73 \mathrm{nmol} /$ day (56-103 nmol/ day), respectively.

Upon inquiry, this volunteer reported to have spent several days a week near open fires, in public smoking areas, and/or in the presence of smokers. Urinary levels of cotinine (mean of $345 \pm 145 \mathrm{nmol} /$ day) and CYMA (mean of $43 \pm 16 \mathrm{nmol} /$ day) were the highest monitored in the whole group. No relation between dietary AA intake and excretion of AAMA and GAMA was observed.

As can be seen in Fig. 3a, some other volunteers also showed inadvertently enhanced CYMA excretions on a distinct number of days (e.g., omnivore-males-2 and -3 and omnivore-female-5). Probably as a consequence of exposure prior to study onset, highest values were recorded on day 1. CYMA excretions of vegan volunteers in general were lower $(0.6 \pm 0.3 \mathrm{nmol} / \mathrm{day}$, under exclusion of veganfemale-4) as compared to those of omnivore volunteers $(2.3 \pm 2.3 \mathrm{nmol} / \mathrm{day})$. Similarly, cotinine values (Fig. $3 \mathrm{~b})$ were $1.8 \pm 5.1 \mathrm{nmol} / \mathrm{day}$ for vegans (excluding veganfemale-4) compared to $39 \pm 157 \mathrm{nmol} /$ day for omnivore volunteers, indicating some exposure to tobacco smoke (presumably second hand). Also see supporting information $\mathrm{S} 5 / 6$.

\section{Discussion}

The aim of this study was to generate scientific data on the impact of consumer preferences on human exposure to foodborne AA. Representative aliquots of duplicates of meals as consumed were obtained to compare dietary exposure of vegan and omnivore volunteers without dietary restriction. Volunteers kept to their accustomed daily habits and provided activity protocols retrospectively. Since unexpectedly enhanced AAMA and GAMA excretions were measured 


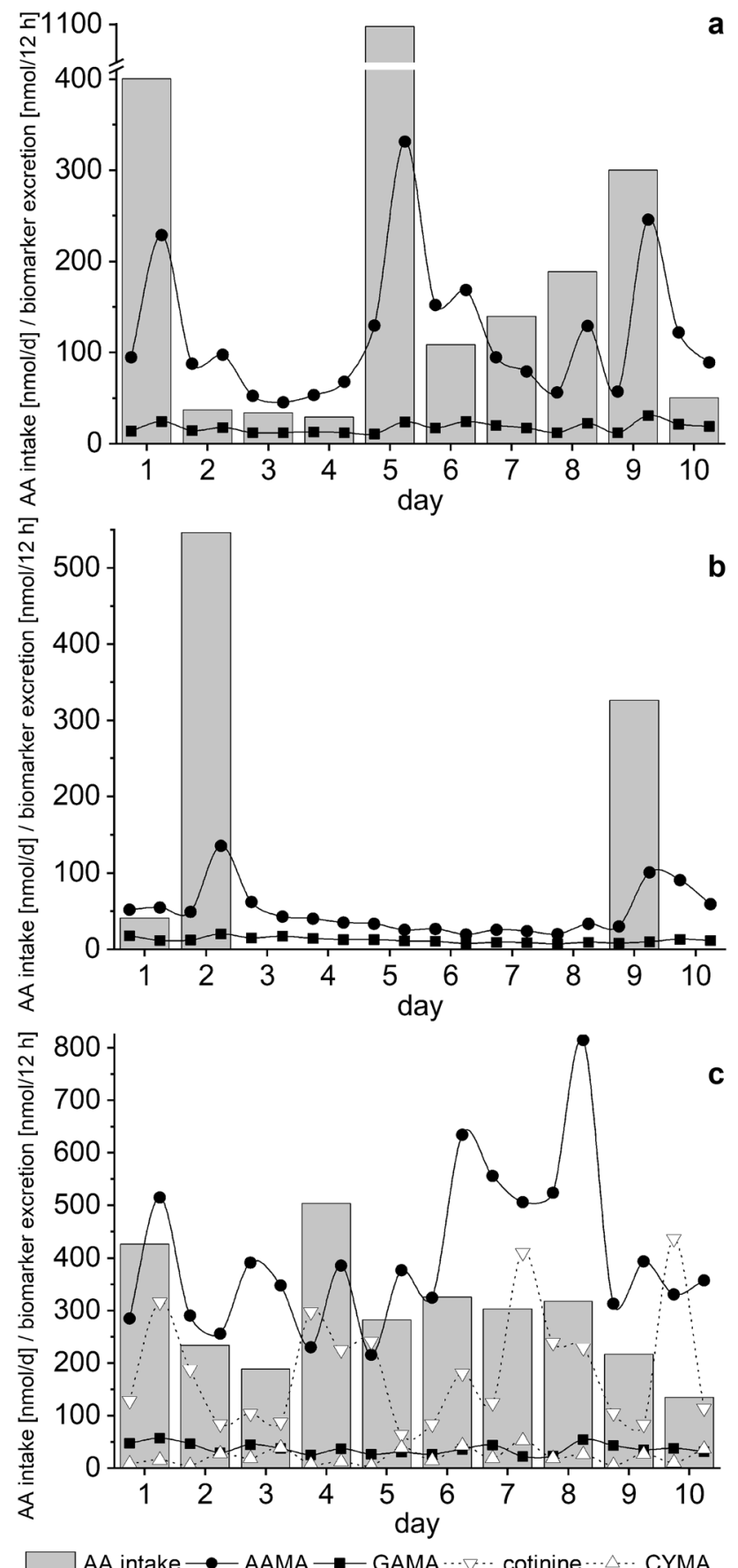

Fig. 2 Acrylamide (AA) intake and excretion kinetics of mercapturic acids and cotinine of a omnivore-female- 1 , b vegan-female- 3 and $\mathbf{c}$ vegan-female-4. CYMA and cotinine levels are only displayed in c. AAMA: $N$-acetyl- $S$-(2-carbamoylethyl)-L-cysteine, CYMA $N$-acetyl$S$-(2-cyanoethyl)-L-cysteine GAMA: $N$-acetyl- $S$-(2-hydroxy-2carbamoylethyl)-L-cysteine

in some volunteers, despite their relatively low dietary AA intake, potential inadvertent exposure to open fire and/or tobacco smoke was suspected. Therefore, in a subgroup $(16 / 20)$ consenting to the respective study amendment, urinary CYMA as well as cotinine levels was monitored. Mean AA intake of all volunteers was estimated to be $0.32 \mu \mathrm{g} / \mathrm{kg}$ bw/day, thus being in the same range as studies from other countries had shown before (Normandin et al. 2013; Sirot et al. 2012).

Although the volunteer collective was small $(n=20)$, results allow some conclusions on the dependency of individual AA dietary exposure on factors of lifestyle and dietary preferences (see Fig. 4). Consumption of deep-fried potato products like French fries led to enhanced dietary AA intake and urinary exposure biomarker (AAMA) excretion in all volunteers. Sustained daily consumption of heatprocessed food such as meat or tofu-based products, of bread or bakery products contributed substantially to overall AA intake. Coffee consumption accounted only for some minor contribution. However, consumption of pan-fried vegetables and meat surrogates like tofu or seitan as well as bread-based products appeared to substantially contribute to enhanced AA intake of the vegan volunteers. For omnivore volunteers, meals with meat content such as burger and casserole with bakery products or biscuits provided a major share to the dietary AA exposure. In a 2-day duplicate diet study from Japan mixed dishes, confectionary, vegetables and beverages were reported to mainly contribute to dietary AA uptake, in some agreement with our results (Yamamoto et al. 2018).

Monitoring the urinary mercapturic acids AAMA and GAMA, informed about the diet-related AA exposure. Excretion of both metabolites essentially was concordant with the AA intake estimated by the described duplicate diet dosimetry. However, incidental exposure to combustion associated external AA sources like open fire and/or tobacco smoke (second hand) supposedly was responsible for unexpectedly high biomarker response. In a vegan volunteer this was detected by monitoring cotinine and CYMA. Goniewicz et al. (2017) recorded almost unchanged urinary cotinine levels in volunteers after switching to nicotine containing e-cigarette vapor inhalation as compared to tobaccobased cigarette smoke. In contrast, levels of the acrylonitrile-related urinary biomarker CYMA were found strongly reduced in the urine of e-cigarette vapor exposed volunteers, in line with further combustion products of organic material, such as polycyclic aromatic hydrocarbons or carbon monoxide. Therefore, CYMA may be considered a bona fide biomarker of exposure to smoke from organic matter forming acrylonitrile during incineration.

Another female vegan (see Fig. 2b) showed excretion kinetics obviously undisturbed by smoke exposure, comparable to those also observed previously in human intervention studies under strictly controlled conditions. These have indicated that an AA intake of $1.3-1.8 \mu \mathrm{g} / \mathrm{kg}$ bw resulted in a mean of $58 \%$ of the ingested AA being excreted as AAMA (7\% as GAMA) within $72 \mathrm{~h}$. (Goempel et al. 2017; Ruenz et al. 2016) In the present study in free-living individuals, 
Fig. 3 Overview of the a CYMA excretion and $\mathbf{b}$ cotinine excretion during the 10-day study for each volunteer. Volunteer vegan-male-5 quit after 9 days, so data were summarized only for 9 days. Boxes, Perc 25/75; whisker, standard deviation; black squares, mean; dash, median; times symbol, minimum; asterisk, maximum; n.q. not quantified (due to no permission)

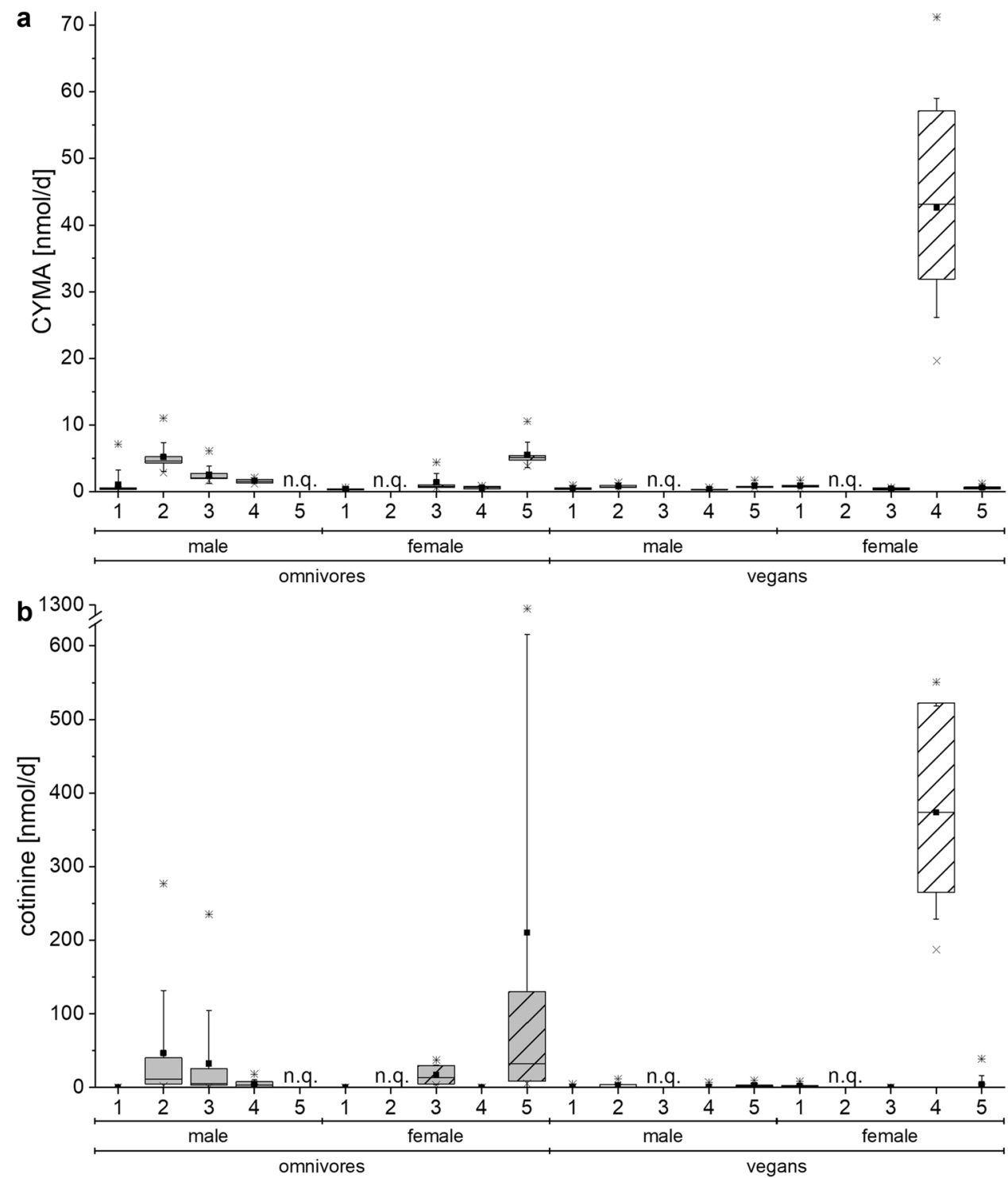

about similar excretion kinetics were seen. AAMA was excreted in less than $72 \mathrm{~h}$ with a maximum value already reached within $12 \mathrm{~h}$ after the reported dietary intake. As case in point, vegan-female-3 excreted within $72 \mathrm{~h}$ about $64 \%$ of the respective dietary AA intake $(546 \mathrm{nmol})$ on day 2 as AAMA. Under strictly controlled conditions, a sustained endogenous background exposure to AA had compellingly been demonstrated in the above studies (Goempel et al. 2017; Ruenz et al. 2016). The present study also supports the premise of a steady background, as exemplified for vegan-female-3. The mean AAMA excretion of this volunteer between days $5-8(51 \pm 5 \mathrm{nmol} / \mathrm{day})$ is estimated to be equivalent to an AA exposure of $0.2 \mu \mathrm{g} / \mathrm{kg}$ bw/day of endogenous origin, assuming $30 \%$ of the respective dose excreted as AAMA within $24 \mathrm{~h}$ (Goempel et al. 2017; Ruenz et al. 2016; Watzek et al. 2012a) This individual endogenous background estimate is close to those observed before
(0.2-0.4 $\mu \mathrm{g} / \mathrm{kg}$ bw/day) (Goempel et al. 2017; Ruenz et al. 2016).

Taken together, the results of this study clearly underline the dependence of exposure to AA on living conditions and dietary preferences. In some cases, vegan lifestyle was found associated with enhanced AA exposure, especially in cases where heat-processed vegan food like meat surrogates were ingested. Although not significant, mean dietary AA exposure of vegan volunteers $(0.38 \mu \mathrm{g} / \mathrm{kg}$ bw/day $)$ exceeded that of omnivore volunteers $(0.26 \mu \mathrm{g} / \mathrm{kg}$ bw/day $)$. In accordance with differential AA exposure vegan volunteers excreted more AAMA (374 nmol/day) and GAMA (50 nmol/day) than the omnivore group (251 nmol/day for AAMA and $40 \mathrm{nmol} /$ day for GAMA). This may well reflect some nutritional preference for thermally treated vegan food.

By comparison, in a study reporting lower frequency of micronuclei in transferrin-positive immature peripheral 


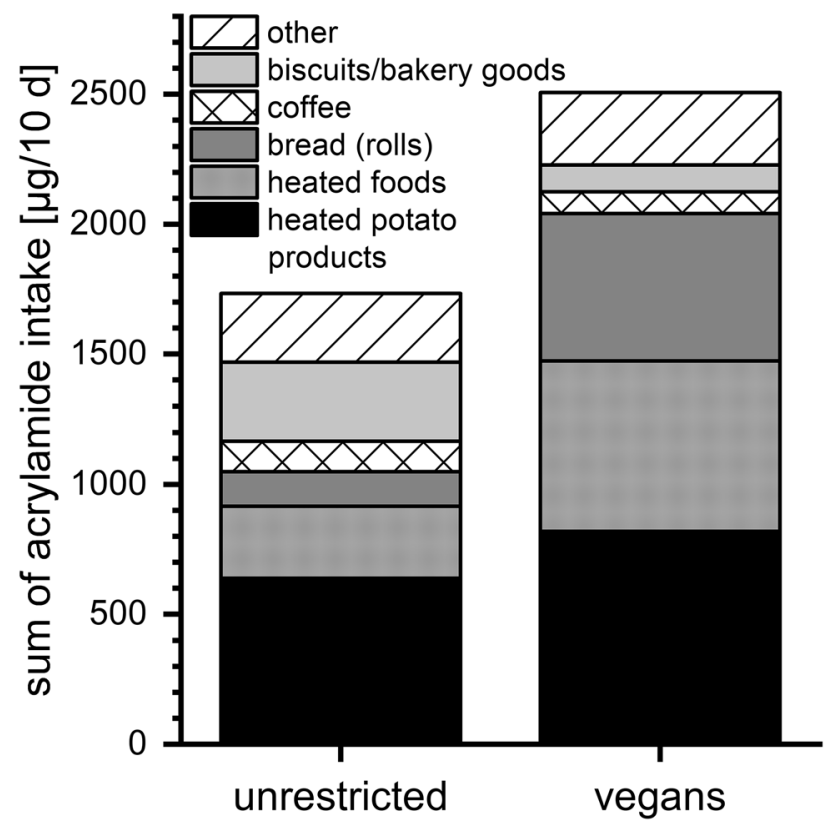

Fig. 4 Comparison of the contribution of different foods to the total acrylamide intake of vegan $(n=10$, one volunteer quit after 9 days) and omnivore $(n=10)$ volunteers following an unrestricted diet, respectively, during the 10-day study

blood reticulocytes of vegetarians (defined by excluding meat and fish from their diet) as compared to non-vegetarians, no significant difference in mean $\mathrm{Hb}$ adduct levels of AA and GA were found (Kotova et al. 2015). The methodology applied in that study is, however, not comparable to our approach. First, there may be differences between vegetarian and vegan nutrition. Second, $\mathrm{Hb}$ adducts are long-term biomarkers of cumulative exposure during the lifetime of human red blood cells, analyzed in that study at one timepoint. In our study, continuous urinary MA monitoring after dietary exposure allows to follow excretion kinetics in detail, thus informing on additional inadvertent exogenous exposure. Duplicate diet studies from Japan considered time intervals of $24 \mathrm{~h}$ and 2 days, respectively, when comparing different methods of AA intake dosimetry (Yamamoto et al. 2018; Kawahara et al. 2019). These short-term dosimetry studies arrived at a somewhat lower range of AA intake as compared to our data, continuously collected over 10 days. Apart from the markedly differential time periods of AA intake monitoring, differing nutritional lifestyles may also have contributed to the observed differences.

The present study has several strengths.

First, the dosimetry of dietary AA intake by analyzing volunteer-prepared dietary duplicates reflects as closely as possible, daily meal consumption within a timeframe of 10 days. A second strength consists of the individual and time-resolved resolution of AA intake and urinary excretion kinetics of exposure biomarkers during the whole study period which allowed to conclude on additional, non-dietary AA exposure sources. Third, passive exposure to sources of environmental smoke from barbecuing, campfires and/or second-hand tobacco smoke was detected by monitoring the respective biomarkers, urinary CYMA and cotinine.

There are, however, also limitations.

First, the study was uncontrolled and it had only a small number of volunteers. Second, analytical findings largely depend on the quality and exactness of dietary duplicate preparation. Although volunteers were trained to prepare representative meal duplicates this may have resulted in somewhat increased variability. Third, depending on the study amendment, consenting volunteers were required to recall and document their then living circumstances (after about 1 year). Fourth, since we had to apply for an amendment to also monitor CYMA and cotinine, not all volunteers originally participating could be contacted or responded, thus reducing participant number with regard to evaluating the specific aspect of (inadvertent) smoke exposure. In addition, urinary levels found for cotinine and CYMA may somewhat deviate from true values, since, in contrast to those for AAMA and GAMA, they were quantified by external standard addition after sample work-up. However, since the respective recovery rates were between 77-99\%, this is considered a minor source of variability, not essentially compromising the overall outcome.

Taken together, this 10-day monitoring study provides new insights into human AA exposure resulting from individual dietary preferences and lifestyles as well as giving further support to ascertainment of endogenous AA exposure. These results are novel and appear of value to pave the way for the future design of related larger scale studies.

Acknowledgements This study has been partly supported by the Federal Institute for Risk Assessment (BfR), Max-Dohrn-Straße 8-10, 10589 Berlin (Grant no. 68561). We thank our volunteers for their participation in the study, Dr. Jens Galan for conducting the study, the members of the research group for supporting the study, and R. Müller, C. Friedland, J. Rotermund, S. Abel, and M. Lieberum for supporting laboratory work.

\section{Compliance with ethical standards}

Conflict of interest The authors declare no conflict of interest.

Ethical standards This human study, approved with two amendments [Ethics Commission of Rhineland-Palatinate, Mainz, No. 837.029.15 (9797)] was performed in accordance with the ethical standards laid down in the 1964 Declaration of Helsinki and its later amendments.

OpenAccess This article is distributed under the terms of the Creative Commons Attribution 4.0 International License (http://creativeco mmons.org/licenses/by/4.0/), which permits unrestricted use, distribution, and reproduction in any medium, provided you give appropriate credit to the original author(s) and the source, provide a link to the Creative Commons license, and indicate if changes were made. 


\section{References}

Boettcher M, Bolt H, Drexler H, Angerer J (2006) Excretion of mercapturic acids of acrylamide and glycidamide in human urine after single oral administration of deuterium-labelled acrylamide. Arch Toxicol 80(2):55-61. https://doi.org/10.1007/s00204-005-0011-y

Bramer SL, Kallungal BA (2003) Clinical considerations in study designs that use cotinine as a biomarker. Biomarkers 8(3-4):187203. https://doi.org/10.1080/13547500310012545

EFSA (2015) Scientific opinion on acrylamide in food. EFSA J 13(6):321. https://doi.org/10.2903/j.efsa.2015.4104

Fennell TR, Sumner SCJ, Snyder RW, Burgess J, Spicer R, Bridson WE, Friedman MA (2005) Metabolism and hemoglobin adduct formation of acrylamide in humans. Toxicol Sci 85(1):447-459

Goempel K, Tedsen L, Ruenz M, Bakuradze T, Schipp D, Galan J, Eisenbrand G, Richling E (2017) Biomarker monitoring of controlled dietary acrylamide exposure indicates consistent human endogenous background. Arch Toxicol 91:3551-3560. https://doi. org/10.1007/s00204-017-1990-1

Goniewicz ML, Gawron M, Smith DM, Peng M, Jacob P, Benowitz NL (2017) Exposure to nicotine and selected toxicants in cigarette smokers who switched to electronic cigarettes: a longitudinal within-subjects observational study. Nicotine Tob Res 19(2):160 167. https://doi.org/10.1093/ntr/ntw160

Jackson LS, Al-Taher F (2005) Effects of consumer food preparation on acrylamide formation. Adv Exp Med Biol 561:447-465. https ://doi.org/10.1007/0-387-24980-X_34

Jakubowski M, Linhart I, Pielas G, Kopecký J (1987) 2-Cyanoethylmercapturic acid (CEMA) in the urine as a possible indicator of exposure to acrylonitrile. Br J Ind Med 44(12):834-840

Kawahara J, Zheng Y, Terui M, Shinohara A, Uyama K, Yoneyama M, Nakajima D, Shibata Y, Adachi S (2019) Dietary exposure to acrylamide in a group of Japanese adults based on 24-hour duplicate diet samples. Food Addit Contam Part A Chem Anal Control Expo Risk Assess 36:15-25. https://doi.org/10.1080/19440 049.2018.1555378

Kotova N, Frostne C, Abramsson-Zetterberg L, Tareke E, Bergman R, Haghdoost S, Paulsson B, Törnqvist M, Segerbäck D, Jenssen D, Grawé J (2015) Differences in micronucleus frequency and acrylamide adduct levels with hemoglobin between vegetarians and non-vegetarians. Eur J Nutr 54(7):1181-1190. https://doi. org/10.1007/s00394-014-0796-7

Minet E, Cheung F, Errington G, Sterz K, Scherer G (2011) Urinary excretion of the acrylonitrile metabolite 2-cyanoethylmercapturic acid is correlated with a variety of biomarkers of tobacco smoke exposure and consumption. Biomarkers 16(1):89-96

Mojska H, Gielecińska I, Cendrowski A (2016) Acrylamide content in cigarette mainstream smoke and estimation of exposure to acrylamide from tobacco smoke in Poland. Ann Agric Environ Med 23(3):456-461. https://doi.org/10.5604/12321966.1219187
Mottram DS, Wedzicha BL, Dodson AT (2002) Acrylamide is formed in the Maillard reaction. Nature 419:448. https://doi. org/10.1038/419448a

Normandin L, Bouchard M, Ayotte P, Blanchet C, Becalski A, Bonvalot Y, Phaneuf D, Lapointe C, Gagné M, Courteau M (2013) Dietary exposure to acrylamide in adolescents from a Canadian urban center. Food Chem Toxicol 57:75-83. https://doi.org/10.1016/j. fct.2013.03.005

Ruenz M, Bakuradze T, Eisenbrand G, Richling E (2016) Monitoring urinary mercapturic acids as biomarkers of human dietary exposure to acrylamide in combination with acrylamide uptake assessment based on duplicate diets. Arch Toxicol 90(4):873-881. https://doi.org/10.1007/s00204-015-1494-9

Rydberg P, Eriksson S, Tareke E, Karlsson P, Ehrenberg L, Törnqvist M (2003) Investigations of factors that influence the acrylamide content of heated foodstuffs. J Agric Food Chem 51(24):70127018. https://doi.org/10.1021/jf034649

Sirot V, Hommet F, Tard A, Leblanc J-C (2012) Dietary acrylamide exposure of the French population: results of the second French Total Diet Study. Food Chem Toxicol 50(3-4):889-894. https:// doi.org/10.1016/j.fct.2011.12.033

Vikström AC, Warholm M, Paulsson B, Axmon A, Wirfält E, Törnqvist M (2012) Hemoglobin adducts as a measure of variations in exposure to acrylamide in food and comparison to questionnaire data. Food Chem Toxicol 50(7):2531-2539. https://doi. org/10.1016/j.fct.2012.04.004

Watzek N, Böhm N, Feld J, Scherbl D, Berger F, Merz KH, Lampen A, Reemtsma T, Tannenbaum SR, Skipper PL (2012a) N 7-Glycidamide-guanine DNA adduct formation by orally ingested acrylamide in rats: a dose-response study encompassing human diet-related exposure levels. Chem Res Toxicol 25(2):381-390

Watzek N, Scherbl D, Feld J, Berger F, Doroshyenko O, Fuhr U, Tomalik-Scharte D, Baum M, Eisenbrand G, Richling E (2012b) Profiling of mercapturic acids of acrolein and acrylamide in human urine after consumption of potato crisps. Mol Nutr Food Res 56(12):1825-1837. https://doi.org/10.1002/mnfr.201200323

Yamamoto J, Ishihara J, Kotemori A, Nakadate M, Sobue T (2018) Validity of estimated acrylamide intake by the dietary record method and food frequency questionnaire in comparison with a duplicate method: a pilot study. J Nutr Sci Vitaminol 64(5):340 346. https://doi.org/10.3177/jnsv.64.340

Zyzak DV, Sanders RA, Stojanovic M, Tallmadge DH, Eberhart BL, Ewald DK, Gruber DC, Morsch TR, Strothers MA, Rizzi GP, Villagran MD (2003) Acrylamide formation mechanism in heated foods. J Agric Food Chem 51(16):4782-4787

Publisher's Note Springer Nature remains neutral with regard to jurisdictional claims in published maps and institutional affiliations. 